\title{
Percepção dos idosos sobre as aulas online de atividade física ofertadas por um projeto extensionista durante a pandemia
}

\author{
Elderly perception about online physical activities \\ classes offered by an extensionist project during \\ the pandemic
}

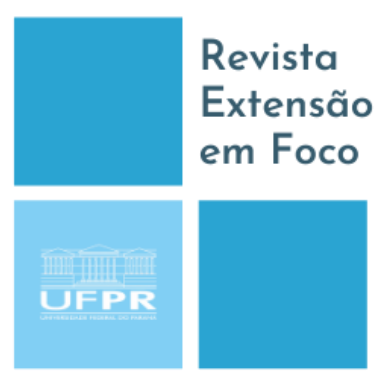

ISSN $2358-7180$

Maria Fernanda Repula Tozetto ${ }^{1}$, Lucélia Justino Borges²

\begin{abstract}
RESUMO
Objetivou-se avaliar a percepção dos idosos sobre as aulas online de atividade física ofertadas pelo Projeto EnvelheSendo/UFPR durante a pandemia da COVID-19. A amostra foi composta por 10 idosas, com idade de 64 a 74 anos. Os dados foram coletados por formulário eletrônico contendo 12 questões sobre dificuldades, conhecimento sobre o aplicativo e se tinha necessidade de ajuda para acesso às aulas online, além de perguntas sobre a percepção das aulas online (benefícios, pontos positivos, dificuldades e preocupações). Foi utilizada análise descritiva e de conteúdo. Os resultados obtidos indicaram que cinco idosas já conheciam o aplicativo e a maioria não tinha dificuldades com o acesso às aulas. A prática de exercício físico foi apontada como o principal motivo da procura pelo Projeto $(n=9)$, além da socialização $(n=7)$, prazer e qualidade de vida $(n=3)$. Quatro idosas relataram que não houve preocupação quanto à mudança do formato das aulas, porém dentre as preocupações foi indicado as possíveis dúvidas em realizar os exercícios $(\mathrm{n}=2)$, falta de contato com os colegas $(\mathrm{n}=2)$, dentre outros. Apesar de três idosas relataram ausência de dificuldade para realizar as aulas online, dentre aquelas que indicaram dificuldades foi citada instabilidade da internet $(n=3)$, horário das aulas $(n=2)$ e realização de trabalhos domésticos/cuidado com familiares $(n=2)$. Dentre os benefícios de realizar as aulas online foi indicado manutenção da rotina de exercícios $(\mathrm{n}=6)$, melhora das capacidades funcionais $(n=4)$ e socialização $(n=3)$. Quanto aos pontos positivos das aulas online foi destacado a praticidade/economia $(n=6)$, socialização $(n=4)$, manter-se ativo durante o isolamento $(n=4)$ e continuar realizando exercícios $(n=3)$. Conclui-se que a percepção das idosas sobre as aulas online foi positiva, considerando o relato de benefícios importantes para o bem-estar, saúde e interação social (virtual) durante a pandemia.
\end{abstract}

Palavras-chave: Idoso. Atividade Motora. Inclusão Digital. Envelhecimento. Exercício Físico.

\section{ABSTRACT}

The objective was to evaluate the perception of the elderly about the online physical activity classes offered by the EnvelheSendo / UFPR Project during the COVID-19 pandemic. The sample consisted of 10 elderly women, aged 64 to 74 years. The data was collected using an electronic form containing 12 questions about difficulties, knowledge about the application and whether help was needed to access online classes, in addition to questions about the perception of online classes (benefits, strengths, difficulties and concerns). Descriptive and content analysis was used. The results obtained indicated that five elderly women already knew the application and the majority had no difficulties with access to classes. The practice of physical exercise was identified as the main reason for looking for the Project $(n=9)$, in addition to socialization $(n=7)$, pleasure and quality of life $(n=3)$. Four elderly women reported that there was no concern about changing the format of the classes, but among the concerns,

${ }^{1}$ Graduanda em Educação Física. Universidade Federal do Paraná (UFPR), Curitiba, Paraná, Brasil. Email: mariatozetto@ufpr.br. Orcid: https://orcid.org/0000-0002-3018-2461

${ }^{2}$ Doutora em Educação Física. Universidade Federal do Paraná (UFPR), Curitiba, Paraná, Brasil. E-mail: lucelia.borges@ufpr.br Orcid: https://orcid.org/0000-0002-0142-3641 
possible questions about performing the exercises $(n=2)$, lack of contact with colleagues $(n=2)$, among others, were indicated. Despite the fact that three elderly women reported the absence of difficulty in conducting online classes, among those who indicated difficulties, internet instability $(n=3)$, class schedule $(n=2)$ and carrying out housework / caring for family members $(n=2)$. Among the benefits of taking the online classes, maintenance of the exercise routine was indicated $(n=6)$, improvement of functional capabilities $(n=4)$ and socialization $(n=3)$. As for the positive aspects of online classes, practicality / economy $(n=6)$, socialization $(n=4)$, staying active during isolation $(n=4)$ and continuing to exercise $(n=3)$ were highlighted. It was concluded that the elderly's perception of online classes was positive, considering the report of important benefits for well-being, health and social (virtual) interaction during the pandemic.

Keywords: Aged. Motor activity. Digital Inclusion. Aging. Exercicse.

\section{INTRODUÇÃO}

O Brasil possui uma população de mais de 200 milhões de pessoas, sendo 10,15\% representado por idosos (IBGE, 2021). O Instituto Brasileiro de Geografia e Estatística (IBGE) vem mostrando que a população de idosos no Brasil tende a crescer significativamente nos próximos anos, chegando em 25,49\% no ano de 2060. (IBGE, 2021).

Em razão disso, políticas públicas que assegurem a participação, saúde, segurança, autonomia e inclusão da população idosa junto a sociedade tornam-se essenciais (WHO, 2005; BRASIL, 2010; BRASIL, 2005). No Brasil, em 1994 foi sancionada a lei $\mathrm{n}^{\mathrm{o}} 8842$, que trata da Política Nacional do Idoso. Esta tem por objetivo assegurar os direitos sociais dos idosos, criando condições para promover sua autonomia, integração e participação efetiva na sociedade. (BRASIL, 2010). Em 1999 foi criada a Política Nacional de Saúde do Idoso (PNSI) pelo Ministério da Saúde (MS). A PNSI visa a promoção do envelhecimento saudável, a prevenção de doenças, a recuperação da saúde e a preservação/melhoria/reabilitação da capacidade funcional dos idosos (BRASIL, 2006; VERAS; OLIVEIRA, 2018). Já em Outubro de 2003 foi instituído o Estatuto do Idoso, lei $\mathrm{n}^{\mathrm{o}}$ 10.741, o qual visa regulamentar os direitos assegurados aos idosos, ou seja, pessoas com 60 anos ou mais. (BRASIL, 2003).

Assim, tais políticas contribuem para assegurar os direitos dos idosos e diversas ações podem ser implementadas a fim de garantir a participação dos idosos na sociedade, para que possam desempenhar suas atividades de maneira independente (VERAS; OLIVEIRA, 2018), com saúde e segurança (BRASIL, 2005). 
Em razão disso, programas para a inclusão do idoso na sociedade tornam-se fundamentais para contribuir com o envelhecimento saudável, seja por meio de grupos de convivência, atividades culturais, de aproximação com a tecnologia ou programas de atividade física, por exemplo. A inovação tecnológica pode contribuir com acesso às informações e aos serviços para a população idosa, oportunizando a inclusão (LIMACOSTA; VERAS, 2003). A literatura tem evidenciado também a importância dos programas de atividade física para os idosos, apontando os diversos benefícios da prática, como a redução/manutenção da gordura corporal, melhoria da flexibilidade, manutenção da massa muscular, da força e a densidade óssea entre outros benefícios físicos, sociais e psicológicos (AZEVEDO FILHO et al., 2018; BORGES, 2014). A prática de atividade física regular também melhora o aspecto emocional com diminuição dos casos de ansiedade e depressão nos idosos (AZEVEDO FILHO et al., 2018).

A busca da atividade física pelos idosos envolve diversos motivos, como recomendação médica, melhoria da saúde, bem-estar, socialização e melhora das capacidades funcionais (BORGES, 2014; AZEVEDO FILHO et al., 2018). Vale ressaltar a importância da atividade física durante toda a vida para quando se chegar à velhice evitar o risco de quedas, limitações físicas e falta de disposição, principalmente, devido a inatividade física na juventude. (RIBEIRO et al., 2016).

No ano de 2020, em decorrência da pandemia da COVID-19, algumas medidas sanitárias foram adotadas, como o distanciamento social e o acesso restrito à áreas públicas e centros esportivos, dificultando o acesso à atividade física. Com isso, a inatividade física e o comportamento sedentário (maior tempo sentado) aumentaram drasticamente no período de isolamento, e pessoas com maior idade, excesso de peso e fisicamente inativas antes da pandemia foram as mais afetadas. (BOTERO et al., 2021).

O estudo conduzido por Malta e colaboradores (2021) investigou que a prática de atividade física diminuiu (28,7\% para $15,74 \%)$ entre os adolescentes e com isso o comportamento sedentário aumentou drasticamente $(44,57 \%$ para $70,15 \%)$ durante o isolamento social (MALTA et al., 2021).

Já no estudo de Brito e colaboradores (2021) foram investigadas as mudanças na atividade física, no sono e na alimentação de 135 pessoas com mais de 18 anos (30\% da amostra avaliada tinha entre 51 e 60 anos) e foi evidenciado que nos primeiros meses da 
pandemia os participantes permaneceram ativos. Concluiu-se também que esse fato deuse pela procura de acompanhamento profissional para realização dos exercícios/treinos pela população avaliada. (BRITO et al., 2021).

Assim, em decorrência do isolamento social, os profissionais da educação física tiveram que realizar mudanças nas metodologias e aplicação dos treinos. A realização/aplicação de exercício físico de forma online vem crescendo muito neste período da pandemia da COVID-19, sendo que os aplicativos de plataformas WhatsApp e Zoom estão entre os mais utilizados, seguidos de aplicativos para prescrição de treinos, como o Mfit e planilhas do Excel (GUIMARÃES et al., 2020). Vale ressaltar que no estudo de Guimarães e col. (2020), os idosos foram o segundo maior grupo que procurou profissionais de educação física na pandemia ou continuaram com a prática com profissionais mesmo de forma remota.

Nesse sentido, a utilização da internet para a prática de atividades físicas pelos idosos só é possível devido à inclusão digital, que vem crescendo com o passar dos anos com a abertura de cursos e oficinas para os idosos (LINDÔSO et al., 2011), contribuindo para o aumento do número de idosos que utilizam a internet (CETIC.BR; 2020). Pesquisas realizadas pelo Centro de Estudos sobre as Tecnologias da Informação e da Comunicação (CETIC) mostraram que, em 2012, apenas 8\% dos idosos brasileiros utilizavam a internet, enquanto em 2019, este percentual aumentou para 34\% (CETIC.BR, 2020). A inclusão digital mostra-se importante, pois permite inserir os idosos no mundo contemporâneo promovendo interação social, autonomia, qualidade de vida e agora, em decorrência da pandemia da COVID-19, aumentar a comunicação entre amigos e familiares para este grupo (DINIZ et al., 2020).

Nesse contexto, em virtude das medidas sanitárias adotadas em decorrência da pandemia da COVID-19, as aulas do Projeto EnvelheSendo da UFPR sofreram alteração para o formato online, desde abril de 2020. O EnvelheSendo é uma ação extensionista do Departamento de Educação Física da Universidade Federal do Paraná (UFPR), que ocorre desde 2016 e tem por objetivo promover o envelhecimento saudável de idosos de Curitiba e Região Metropolitana, por meio da oferta de atividades físicas e socioculturais. Deste modo, avaliar a percepção dos idosos relacionado a esta mudança no formato de aula do Projeto se torna importante, pois favorece identificar os pontos positivos e negativos dessa mudança, bem como oportunizará ajustes para o desenvolvimento das aulas, uma vez que as medidas sanitárias da COVID-19 continuam 
em vigor. Além disso, os resultados contribuirão com a produção do conhecimento sobre inclusão digital dos idosos e atividade física, bem como poderá auxiliar no desenvolvimento de outras ações e projetos voltados a este grupo etário, que tem crescido de forma exponencial no Brasil.

À vista do exposto, esse estudo tem por objetivo investigar a percepção dos idosos sobre as aulas online de atividade física ofertadas por um projeto extensionista durante a pandemia da COVID-19.

\section{MÉTODO}

Trata-se de um estudo descritivo e transversal realizado com idosos participantes do projeto extensionista EnvelheSendo da UFPR.

A composição da amostra foi intencional e não probabilística. Os critérios de inclusão para composição da amostra foram: ter 60 anos ou mais, ambos os sexos, ter realizado as aulas de modo presencial (antes da pandemia) e de forma online (durante a pandemia) e aceitar o convite para participar da pesquisa. Os critérios de exclusão foram: a não participação nas aulas online identificada pela lista de frequência e ser aluno novato (não ter realizado as aulas presenciais). Um participante do sexo masculino foi excluído por não ter 60 anos. Assim, a amostra foi composta por 10 idosas, com idade entre 64 a 74 anos.

A pesquisa foi realizada de forma online respeitando as medidas sanitárias adotadas para o enfrentamento da pandemia da COVID-19. Os idosos que aceitaram participar da pesquisa, após a leitura do Termo de Consentimento Livre e Esclarecido (TCLE) assinalaram a opção "Eu concordo voluntariamente em participar deste estudo", do formulário online (Formulário Google) e tiveram acesso ao restante do formulário.

$\mathrm{O}$ instrumento de pesquisa foi aplicado no formato de questionário pelo formulário online. O questionário continha 12 perguntas (abertas e fechadas), o qual foi elaborado pela pesquisadora principal pautando-se no objetivo proposto e no referencial teórico da área.

As questões fechadas envolveram: a) variáveis sociodemográficas (sexo e idade); b) tempo de participação no projeto (1, 2, 3 ou 4 anos ou mais); c) dificuldades em realizar as aulas online (espaço para realizar as aulas em casa, dificuldade em acessar o 
aplicativo das aulas online, falta de material para realizar os exercícios, horário das aulas, instabilidade da conexão da internet, falta de tempo, trabalhos domésticos e/ou cuidados com os familiares, falta de disposição em realizar a aula em casa, problemas de saúde (doenças) e outros, podendo marcar mais de uma opção de resposta; d) se tinha conhecimento sobre o aplicativo utilizado para as aulas online (sim, não ou pouco); e) se tinha necessidade de ajuda de terceiro para acessar o aplicativo utilizado para as aulas (sim, não ou às vezes).

Já as perguntas abertas envolveram: a) avaliação da percepção dos idosos com a mudança do modelo de aula, sobre as preocupações, motivação para continuar e benefícios percebidos (presencial para o remoto); b) motivos pelo qual escolheu participar do Projeto (antes da pandemia); c) dificuldades em realizar/acessar as aulas; d) pontos positivos de se ter as aulas online; e) outros comentários sobre a percepção dos idosos em relação às aulas online.

Dentre os procedimentos para a coleta de dados, destaca-se que o convite com o respectivo link do formulário foi enviado aos idosos por meio do aplicativo WhatsApp, considerando os contatos telefônicos de cadastro no Projeto. O período para resposta dos idosos foi pré-determinado em uma semana. Após quatro dias, foi realizado novo contato para relembrar o convite da pesquisa, porém de forma verbal durante a aula do Projeto. No último dia da coleta foi realizado mais um contato por meio do aplicativo WhatsApp, relembrando o convite para participar da pesquisa. A coleta de dados ocorreu em março de 2021.

A análise dos dados foi realizada de forma descritiva (frequência absoluta, desvio padrão e média) e pela análise de conteúdo. $\mathrm{O}$ projeto de pesquisa atendeu preceitos éticos. O convite com a explicação geral dos objetivos da pesquisa e dos procedimentos a serem realizados foi enviado aos participantes. Aqueles que aceitaram participar da pesquisa, tiveram acesso ao Termo de Consentimento Livre e Esclarecido (TCLE) online. Após leitura do TCLE e concordância, assinalaram a opção "Eu concordo voluntariamente em participar deste estudo". No TCLE foi garantida a confidencialidade das informações, os riscos mínimos e os benefícios da pesquisa, a participação voluntária, a possibilidade de deixar o estudo a qualquer momento sem necessidade de justificativa, dentre outros aspectos, atendendo à Resolução nº66/2012 do Conselho Nacional de Saúde. 


\section{RESULTADOS}

O presente estudo contou com a participação de 10 idosas com a média de idade de 69,2 anos $( \pm 3,2)$. A maioria das investigadas participam do Projeto há três anos $(\mathrm{n}=5)$, sendo a média de participação de 2,9 anos $( \pm 0,54)$.

Em relação à mudança no formato de aula (presencial para online), cinco idosas afirmaram ter conhecimento sobre os aplicativos utilizados, enquanto cinco não os conheciam antes de utilizá-los para a prática das aulas. Foi apontado também por sete idosas que estas não necessitavam de ajuda para acessar as aulas e apenas três marcaram que "às vezes" necessitavam de ajuda para o acesso.

Foi observado que a procura por exercício físico $(n=9)$ é o principal motivo que levou as idosas a procurarem as aulas do projeto quando eram realizadas presencialmente. A procura por socialização com pessoas da mesma idade $(n=7)$, prazer e qualidade de vida $(n=3)$ e a melhora da saúde mental $(n=1)$ também foram motivos citados pelas participantes.

Em relação à mudança no modelo de aula presencial para online foi mais relatado que não houve preocupações $(n=4)$. E as preocupações quando relatadas foram as possíveis dificuldades que teriam em realizar os exercícios $(n=2)$, a falta de contato com os colegas $(n=2)$, dificuldades com o aplicativo/internet $(n=1)$ e dúvidas de como seriam realizadas as aulas $(n=1)$. Alguns comentários das idosas referente às preocupações que tiveram ao saber da mudança no formato das aulas serão apresentados a seguir:

\footnotetext{
"Se conseguiria acompanhar." (Idosa 1).

"Ñ conseguir fazer os exercícios corretamente." (Idosa 2).

"Entendi perfeitamente, [mesmo] sentindo a falta do contato fisico" (Idosa 3)

"Como seriam e como ia participar" (Idosa 4).

"Adoro sair, passear, conversar, viajar. A preocupação é não concentração por longo tempo em usar novas tecnologias." (Idosa 5).
}

Como relatado, algumas idosas não tiveram preocupações ao receber a notícia da mudança no modelo de aula. Alguns comentários em relação a essa questão serão apresentados a seguir:

\footnotetext{
"Fiquei feliz pela continuidade do projeto." (Idosa 6).

"Como estava sem carro, achei a melhor solução" (Idosa 7).

"Fiquei feliz pois não iríamos parar." (Idosa 8).
} 
Quanto à motivação para continuar nas aulas após a mudança de formato (presencial para online), manter-se ativo $(n=7)$ foi o mais apontado pelas idosas, além de socialização $(n=4)$, qualidade das aulas $(n=2)$, saúde/bem-estar $(n=1)$ e manter-se em casa $(n=1)$. As respostas das idosas referente às motivações que levaram-nas a participar das aulas online podem ser observadas a seguir:

"A possibilidade de continuar me exercitando e interagindo com os amigos do projeto." (Idosa 6).

"O desejo de ñ ficar parada" (Idosa 3).

"São aulas importantes pro meu bem estar." (Idosa 2).

"Os exercícios. Muito importantes." (Idosa 1).

No que diz respeito às dificuldades em realizar as aulas online, três participantes afirmaram não ter nenhuma dificuldade $(n=3)$, enquanto a instabilidade com a internet $(\mathrm{n}=3)$, o horário das aulas $(\mathrm{n}=2)$, trabalhos domésticos e/ou cuidado com familiares $(n=2)$, espaço para realizar as aulas em casa $(n=1)$, falta de tempo $(n=1)$, falta de disposição para realizar aulas em casa $(n=1)$ e problemas de saúde $(n=1)$ foram as dificuldades enfrentadas pelas idosas na realização das aulas online. Vale ressaltar que para esta pergunta poderia ser assinalada mais de uma opção de resposta.

As idosas relataram que a praticidade/economia $(n=5)$ foi o principal ponto positivo de ter as aulas de forma online. Manter-se ativo respeitando o isolamento $(n=4)$, socialização $(n=4)$, continuar realizando exercício físico $(n=3)$ e a dedicação dos professores com as aulas $(n=1)$ foram os outros pontos positivos relatados. As respostas para os aspectos positivos sobre a mudança para aulas online podem ser observadas a seguir:

\footnotetext{
"Mantenho minhas atividades sem risco. "(Idosa 2).

"Diversos motivos, entre eles reconhecimento em saber que vcs se dedicam a isso, da maneira mais agradável e prazerosa, nos ensinando e motivando . E assim, vendo as colegas do grupo nos motiva ainda mais..." (Idosa 3).

"Abrange mais pessoas, conforto de fazer estar em casa. Demanda menos tempo custos com transporte e mesmo de forma on LINE nos mantemos contatos pelo menos 2 vezes por semana." (Idosa 9).

"Continuar mantendo contato com os amigos e me exercitando." (Idosa $6)$.
}

Já em relação aos benefícios de continuar realizando as aulas de forma online foi indicado manter a rotina de exercícios $(n=6)$, a melhora das capacidades funcionais $(n=4)$, socialização $(n=3)$, bem-estar/disposição $(n=2)$. As respostas das idosas referente aos benefícios de se ter aulas online podem ser verificadas a seguir: 
"Sou movida a exercicio ( dentro das minhas possibilidades) agora aos 71 anos ja estou com problema de joelho, que em alguns exercicios me atrapalha ...mas sempre foi o bem estar, a continuidade da [força] fisica, equilibrio, etc" (Idosa 3).

"Ânimo de vida, mais disposição física, sozinha não faço exercícios." (Idosa 10).

"Continuar exercitando e me manter em contato com as amizades." (Idosa 1).

A última questão do formulário foi relacionada à participação das idosas nas aulas e a percepção delas sobre as mesmas, sendo aberta a demais comentários. Agradecimentos e elogios às aulas, professores e ao Projeto foi o assunto mais abordado entre eles $(n=6)$. Além disso, também foi destacada a importância de gravar as aulas para poderem ser realizadas quando possível $(n=1)$, socialização $(n=1)$ e a melhora do corpo com as atividades $(\mathrm{n}=1)$.

\footnotetext{
"Eu só tenho a agradecer todo esse tempo ( desde lá do começo ) que participo do Projeto . Visto a camiseta com muito orgulho! Agradeço a UFPR, a prof [....] e a cada um de vocês alunos, quase professores que se dedicam a isso!" (Idosa 3).

"Na minha opinião é super necessária, deveria ser uma atividade emergencial diante do quadro de saúde física e emocional neste período difícil que estamos vivendo. Percebo então que foi muito bem vinda essa iniciativa do Projeto Envelhecendo na hora certa no tempo certo e inovador. Tomada de decisão muito assertiva e com sucesso,notado pela participação ativa de nós os [beneficiários] desse projeto.” (Idosa 9).

"Um agradecimento especial à professora [....]e seus pupilos pela dedicação e carinho que tiveram conosco durante todo esse tempo, sempre se preocupando em elaborar aulas criativas, eficazes e lúdicas. Meu muito obrigada." (Idosa 6).

"Muita dedicação dos participantes deste projeto, coordenação e alunos dedicados e competentes". (Idosa 4).
}

\section{DISCUSSÃO}

No presente estudo, a maioria das idosas relataram que não necessitavam de ajuda para acessar as aulas online e metade delas já tinham conhecimento sobre os aplicativos utilizados, demonstrando acesso à tecnologia e internet, sendo este um dos possíveis motivos da aceitação ao novo formato de aula e consequentemente mostra a percepção positiva das idosas em relação às aulas online. A motivação para o ingresso e continuidade no Projeto com as aulas online envolveu a prática de exercício físico e a socialização. Manter a rotina dos exercícios físicos e com isso a melhora das capacidades funcionais foram os benefícios apontados pelas idosas. Além disso, foi 
destacado como principal ponto positivo a praticidade e economia, devido à ausência de deslocamento até o Departamento de Educação Física da UFPR, que é o local onde as aulas presenciais eram realizadas antes da pandemia da COVID-19. Dentre as preocupações, a maioria das idosas mencionaram não ter preocupações com a mudança do formato das aulas e quando relatadas foram relacionadas à possíveis dúvidas de como seriam as aulas e como realizar os exercícios. A principal dificuldade encontrada para realizar as aulas online foi a instabilidade da internet, horário das aulas e afazeres domésticos/cuidado com familiares.

Levando em consideração as respostas avaliadas pelo presente estudo, nota-se que a inclusão digital está presente neste grupo. Esse fato pode ser justificado pela participação das idosas nos projetos da Universidade Aberta da Maturidade (UAM) / UFPR nos anos anteriores. A UAM também é uma ação extensionista da UFPR que tem como objetivo contribuir para a qualidade de vida e para o desenvolvimento social e cultural dos idosos (UAM, 2021). Os principais conteúdos abordados nos projetos realizados pela UAM são a Inclusão Digital, Direito do Idoso, Saúde do idoso, Atividades Corporais, entre outros (UAM, 2021).

Em um estudo conduzido por Lindôso e colaboradores (2011) foi avaliado a percepção subjetiva de memória, memória lógica e habilidade manual de 51 idosos participantes das Oficinas de Inclusão Digital do Projeto Potencialldade do Instituto de Geriatria e Gerontologia da PUCRS. Os autores verificaram que os idosos que participavam das oficinas apresentaram um resultado satisfatório para a memória e as habilidades manuais, mostrando a importância do projeto (LINDÔNSO et al., 2011).

A internet pode oportunizar benefícios como a autonomia e a independência para os idosos (DINIZ et al., 2020). Estudo realizado com idosos evidenciou que estes utilizam a internet para a socialização, seja com amigos, familiares ou relacionamentos amorosos, e entretenimento, como ver vídeos e fotos, ler e ouvir música (DINIZ et al., 2020). Vale ressaltar que este estudo envolveu $85 \%$ de idosos jovens (menores de 68 anos), fato que preocupa quando se trata dos idosos mais velhos, entretanto foi apontado que a internet também pode ser usada para inclusão deles no mundo contemporâneo. (DINIZ et al., 2020).

Em razão disso, a internet pode favorecer a inclusão dos idosos na sociedade promovendo a socialização (DINIZ et al., 2020). Como relatado pelas idosas do Projeto EnvelheSendo, a socialização é um dos motivos pelo qual elas ingressaram no projeto e também é um dos motivos que auxiliam na permanência nas aulas online. Assim, mesmo sem o contato físico, a interação social é um dos principais benefícios que o projeto oportunizou para o grupo.

Os projetos de convivência para idosos oportunizam diversos benefícios como disposição, autoestima, felicidade, bem-estar, diminuição da sensação de solidão e 
percepção sobre o corpo por meio da atividade física (BIANCHINI et al., 2018). O estudo conduzido por Bianchini e colaboradores (2018) verificou como grupos de convivência influenciam na vida do idoso por meio da prática de atividade física, com relatos sobre estes benefícios e a satisfação dos idosos por fazer parte de um grupo de sua idade. (BIANCHINI et al., 2018).

Krug et al. (2019) realizaram oficinas de inclusão digital para estimulação e reabilitação cognitiva de idosos participantes de um estudo em um projeto de extensão. O programa de inclusão digital era composto por uso de computador, realização de atividade física e novamente uso do computador na mesma sessão. Foram observados benefícios da socialização, aprendizagem do uso de computadores e melhora da aptidão física nos idosos avaliados. (KRUG et al., 2019).

Além da socialização, a busca pela prática de exercício físico também foi evidenciada. Manter o corpo ativo todos os dias, mesmo durante a pandemia, foi destacado como importante para as idosas, fazendo com que se torne também o motivo pela continuação no Projeto e o principal benefício que as aulas online oferecem, demonstrando a importância do Projeto na vida delas. A percepção dos benefícios da atividade física na qualidade de vida e na socialização também foram evidenciados pelos idosos frequentadores dos Pontos de Encontros Comunitários (PECs) do Distrito Federal (AZEVEDO FILHO et al., 2019), mostrando que os PECs têm grande importância na vida dessa população (AZEVEDO FILHO et al., 2019). A pesquisa conduzida por Navarro e col. (2008), também demonstrou a importância da atividade física na vida dos idosos. A pesquisa foi realizada no projeto "Viva Bem a Idade que Você Tem", no qual era realizado atividade física três vezes na semana. Dentre os benefícios apontados pelos idosos, a busca por um estilo de vida mais saudável foi o mais apontado, seguido por indicação médica e interação social. (NAVARRO et al., 2008). Os autores ressaltam a importância dos espaços destinados à realização de exercícios físicos e lazer para adesão da prática pelos idosos (NAVARRO et al., 2008).

A mudança do formato de aula (presencial para online) gerou algumas preocupações, porém estas foram relacionadas à dúvidas sobre como seriam as aulas, fazendo com que elas ainda quisessem participar das mesmas pelo desejo de se manterem ativas durante o isolamento. Das idosas avaliadas, cinco relataram que a praticidade/economia foi um ponto positivo dessa mudança, fato que se dá por conta do difícil acesso ao prédio onde eram realizadas as aulas presenciais antes da pandemia.

Vale ressaltar que estudo realizado com 13 idosas, de 64 a 75 anos, desistentes do Projeto EnvelheSendo mostrou que o principal motivo que levou à desistência das aulas era a locomoção até o local, seguido por problemas de saúde (SANTOS et al., 2020). Considerando que a praticidade/economia é um dos principais pontos positivos de realizar as aulas online, a permanência nas aulas neste novo formato durante a pandemia 
podem ter auxiliado a permanência das mesmas no Projeto. Soma-se a isso a percepção positiva das idosas sobre as aulas online como oportunidade de manterem-se ativas respeitando o isolamento preconizado pelas medidas sanitárias da COVID-19. Foi observado também que no espaço para comentários gerais sobre as aulas online, as idosas fizeram elogios e agradecimentos ao Projeto, às aulas e aos professores, demonstrando a percepção positiva e reconhecendo os benefícios. Em razão disso, mostra-se a importância que o Projeto EnvelheSendo tem na vida dos alunos participantes, promovendo uma melhor qualidade de vida e diversos benefícios biopsicossociais. Os resultados também sinalizam a importância da manutenção destas ações e da implementação de mais projetos acessíveis para esse grupo etário.

Destaca-se como limitação do estudo a participação exclusiva das mulheres na amostra, o que impede a extrapolação dos resultados para os homens. No ano de 2020, quatro homens participaram das aulas online do EnvelheSendo. Além do óbito de um destes participantes, houve a exclusão de um homem com menos de 60 anos. Os outros dois participantes não retornaram sobre o convite da pesquisa. Como aspectos positivos destacam-se a oportunidade de investigar a percepção das idosas sobre a mudança das aulas, para que assim os estudantes e professores possam melhorar cada vez mais a forma de desenvolver as aulas, atendendo melhor os idosos.

\section{CONSIDERAÇÕES FINAIS/CONCLUSÃO}

Os resultados indicam a inclusão digital nas idosas avaliadas, o que pode ter facilitado e contribuído para a aceitação na mudança do formato das aulas do Projeto EnvelheSendo/UFPR (presencial para online) e para a percepção positiva sobre as aulas online. A metade das idosas relatou ter conhecimento sobre os aplicativos utilizados, e a maioria relatou que não necessitava de ajuda para acessar as aulas online, não tinha preocupações com a mudança do formato das aulas nem dificuldades para participar das aulas online. Porém, dentre aquelas que afirmaram ter preocupações, as mesmas foram relacionadas a possíveis dúvidas de como seriam as aulas, como realizariam os exercícios e a falta de contato com os colegas. Dentre as dificuldades foram relatados problemas com a conexão, horários das aulas e problemas com as atividades realizadas em casa, como trabalhos domésticos e/ou cuidado com familiares.

A busca pela prática do exercício físico foi o motivo mais citado pela procura ao Projeto, seguido de socialização e prazer e qualidade de vida. Manter-se ativo, mantendo o contato com os colegas e a qualidade das aulas foram as principais motivações citadas pelas idosas para continuar realizando as aulas. Em relação aos pontos positivos das aulas online, a praticidade/economia foi o mais citado, seguido de manter-se ativo respeitando o isolamento e a socialização. A manutenção da rotina de 
exercícios, a melhora das capacidades funcionais e a socialização foram os benefícios citados ao realizar as aulas online.

Conclui-se que a percepção das idosas sobre às aulas online foi positiva, considerando o relato de benefícios importantes para o bem-estar, saúde e interação social (virtual) durante a pandemia. A mudança no modelo de aula (presencial para online) gerou a necessidade de adaptação, tanto dos alunos idosos quanto dos estudantes e professores envolvidos no projeto extensionista. Algumas dificuldades, preocupações e dúvidas foram relatadas pelas idosas, porém, os benefícios superaram estes aspectos conforme observado nos relatos das idosas. Além disso, foi evidenciado a importância do Projeto na vida das participantes, em especial, neste momento da pandemia da COVID-19 conforme destacado nos diversos elogios e agradecimento às aulas, professores e ao projeto.

A presente investigação oportunizou reflexão sobre pontos importantes, como a inclusão digital e social dos idosos e também a importância da atividade física na vida dos mesmos durante um momento atípico devido à pandemia da COVID-19. Futuros estudos envolvendo também homens idosos e análises pela idade, considerando a diversidade e pluralidade das diferentes gerações envelhecidas, podem contribuir para avaliação da percepção sobre as aulas de atividade física desenvolvidas durante a pandemia da COVID-19.

\section{REFERÊNCIAS}

AZEVEDO FILHO, E. R.; CHARIGLIONE, I. P. F. S.; SILVA, J. T. C.; VALE, A. M. S.; ARAÚJO, E. K. H. S.; SANTOS, M. F. R. The perception of the elderly about the benefits of practicing physical activity: a study at the Community Meeting Points of the Distrito Federal. Revista Brasileira de Ciências do Esporte, v. 41, n. 2, p. 142149, 2019. Disponível em The perception of the elderly about the benefits of practicing physical activity: a study at the Community Meeting Points of the Distrito Federal. Acesso em 25 mar. 2021. https://doi.org/10.1016/j.rbce.2018.04.010

BIANCHINI, F.; LOHMANN, P.; PISSAIA, L.; COSTA A. Percepção de idosos participantes de atividades físicas em grupo de convivência sobre sua saúde, Espaço Ciência e Saúde, Alta Cruz, v. 6, n 2, p. 88-96, dez. 2018. Disponível em http://200.19.0.181/index.php/enfermagem/article/view/8066/1770. Acesso em 8 mar. 2021.

BRASIL. Lei $\mathbf{n}^{\circ}$ 10.741, $^{\circ}$ de outubro de 2003. Instituído o Estatuto do Idoso, Congresso Nacional, Brasília, DF, [2007]. Disponível em http://www.planalto.gov.br/ccivil_03/leis/2003/110.741.htm. Acesso em 25 mar. 2021. 
BRASIL. Lei $\mathbf{n}^{\mathbf{0}}$ 2.528, 19 de outubro de 2006. Aprovar a Política Nacional de Saúde da Pessoa Idosa. Ministério da Saúde, Brasília, DF, [2006]. Disponível em http://bvsms.saude.gov.br/bvs/saudelegis/gm/2006/prt2528_19_10_2006.html. Acesso em 25 mar. 2021.

BRASIL. Lei $n^{\circ}$ 8.842, 4 de janeiro de 1994. Política Nacional do Idoso. Ministério do Desenvolvimento Social e Combate à Fome, Brasília, DF, [2010]. Disponível em http://www.mds.gov.br/webarquivos/publicacao/assistencia_social/Normativas/politica idoso.pdf. Acesso em 25 mar. 2021

BRITO, L. M. S.; LIMA, V. A.; MASCARENHAS, L. P.; MOTA, J.; LEITE, N. PHYSICAL ACTIVITY, EATING HABITS AND SLEEP DURING SOCIAL ISOLATION: FROM YOUNG ADULT TO ELDERLY. Rev Bras Med Esporte, São Paulo, v. 27, n. 1, p. 21-25, Jan. 2021. Disponível em https://www.scielo.br/scielo.php?script=sci_arttext\&pid=S1517-

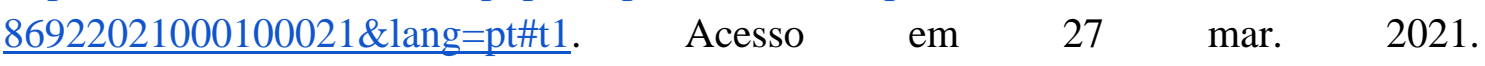
https://doi.org/10.1590/1517-8692202127012020_0061.

BORGES, L. J. SINTOMAS DEPRESSIVOS E ATIVIDADE FÍSICA EM IDOSOS: ESTUDO LONGITUDINAL. Tese (Doutorado em Educação Física) - Centro de Desportos, Universidade Federal de Santa Catarina, Florianópolis, 2014. Disponível em

https://repositorio.ufsc.br/xmlui/bitstream/handle/123456789/129629/328832.pdf?seque $\underline{\text { nce }=1 \& \text { is Allowed }=y}$. Acesso em 26 mar. 2021.

BOTERO, J. P.; FARAH, B. Q.; CORREIA, M. A.; LOFRANO-PRADO, M. C.; CUCATO, G. G.; SHUMATE, G. RITTI-DIAS, R. M.; PRADO, W. L. Impact of the COVID-19 pandemic stay at home order and social isolation on physical activity levels and sedentary behavior in Brazilian adults Einstein (São Paulo), v. 19, p. 1-6, 2021. Disponível em https://www.scielo.br/scielo.php?script=sci_arttext\&pid=S167945082021000100900\&lang=pt. Acesso em 25 mar. 2021. http://dx.doi.org/10.31744/einstein_journal/2021ae6156.

CETIC.BR. TIC Domicílios, Publicações. Disponível em https://cetic.br/pt/pesquisa/domicilios/publicacoes/. Acesso em 26 mar. 2021.

DINIZ, J. L.; MOREIRA, A. C. A.; TEIXEIRA, I. X.; AZEVEDO, S. G. V. A.; FREITAS, C. A. S. L.; MARANGUAPE, I. C. Digital inclusion and Internet use among older adults in Brazil: a cross-sectional study. Rev. Bras. Enferm. Brasília, v. 73, supl. 3, e20200241, 2020. Disponível em https://www.scielo.br/scielo.php?script=sci_arttext\&pid=S0034-

71672020001500184\&lng=pt\&nrm=iso\#B1. Acesso em 27 mar. 2021. Epub 06-Nov2020. https://doi.org/10.1590/0034-7167-2020-0241. 
GUIMARÃES, J. A. C.; GUERRA, P. H.; UENO, D. T.; CHRISTOFOLETTI, A. E. M.; NAKAMURA, P. M. Estudo transversal sobre uso de ferramentas virtuais para orientar a atividade física durante a COVID-19. Revista Brasileira de Atividade Física \& Saúde, $\quad$ v. $25, \quad$ p. 1-8, 2020. Disponível em https://rbafs.org.br/RBAFS/article/view/14410. Acesso em 27 mar. 2021. DOI: $10.12820 /$ rbafs. $25 \mathrm{e} 0150$

IBGE Instituto Brasileiro de Geografia e Estatística. População. Brasil, 2021. Disponível em https://www.ibge.gov.br/apps/populacao/projecao/index.html. Acesso em 11 mar. 2021.

KRUG, R. R.; ONO, L. M.; FIGUEIRÓ, T. H.; XAVIER, A. J.; D’ORSI, E. Programa intergeracional de estimulação cognitiva: Benefícios relatados por idosos e monitores participantes. Psic.: Teor. e Pesq., Brasília, v. 35, 2019. Disponível em https://www.scielo.br/scielo.php?script=sci_arttext\&pid=S0102-

37722019000100405\&lng=pt\&nrm=iso\#B20. Acesso em 8 abr. 2021. http://dx.doi.org/10.1590/0102.3772e3536.

LIMA-COSTAS, M. F.; VERAS, R. Saúde pública e envelhecimento. Cad. Saúde Pública, v. 19, n. 3, p. 700-701, jan. 2003. Disponível em https://www.scielo.br/scielo.php?script=sci_arttext\&pid=S0102-311X2003000300001. Acesso em 11 mar. 2021. https://doi.org/10.1590/S0102-311X2003000300001

LINDOSO, Z. C. L.; CAMMAROTA, M. P.; ARGIMON, I. I. L.; GOMES, I.; SCHWANKE, C. H. A. Percepção subjetiva de memória e habilidade manual em idosos de uma oficina de inclusão digital. Rev. bras. geriatr. gerontol., Rio de Janeiro, v. 14, n. 2, p. 303-317, jun. 2011. Disponível em https://www.scielo.br/scielo.php?script=sci_arttext\&pid=S180998232011000200011\&lng=pt\&nrm=iso. Acesso em 27 mar. 2021. https://doi.org/10.1590/S1809-98232011000200011.

MALTA, D; C.; SZWARCWALD, C. L.; BARROS, M. B. A.; GOMES, C. S.; MACHADO, I. E.; JUNIOR, P. R. B. S.; ROMERO, D. E.; LIMA, M. G.; DAMACENA, G. N.; PINA, M. F.; FREITAS, M. I. F.; WERNECK, A. O.; SILVA, D. R. P.; AZEVEDO, L. O.; GRACIE, R. The COVID-19 Pandemic and changes in adult Brazilian lifestyles: a cross-sectional study. Epidemiol. Serv. Saúde, Brasília, v. 29, n. 4, e2020407, 2020. Disponível em https://www.scielo.br/scielo.php?pid=S2237$96222020000400315 \&$ script=sci_arttext\&tlng=en. Acesso em 29 mar. 2021. https://doi.org/10.1590/s1679-49742020000400026.

NAVARRO, F. M.; RABELO, J. F.; FARIA, S. T.; LOPES, M. C. L.; MARCON, S. S. Percepção de idosos sobre a prática de atividade e importância da atividade física em suas vidas. Revista Gaúcha de Enfermagem, Porto Alegre, v. 29, n. 4, p. 596-603, dez. 2008.

Disponível em 
https://www.seer.ufrgs.br/RevistaGauchadeEnfermagem/article/view/7631/4686. acesso em 13 abr 2021.

RIBEIRO, A. Q.; SALGADO, S. M. L.; GOMES, I. S.; FAGAL, A. S.; MARTINHO, K. O.; ALMEIDA, L. F. F.; OLIVEIRA, W. C. Prevalência e fatores associados à inatividade física em idosos: um estudo de base populacional. Rev. bras. geriatr. gerontol, Rio de Janeiro, v. 19, n. 3, p. 483-493, jun. 2016. Disponível em https://www.scielo.br/scielo.php?script=sci_abstract\&pid=S1809-

98232016000300483\&lng=pt\&nrm=iso\&tlng=pt. Acesso em 26 mar. 2021. https://doi.org/10.1590/1809-98232016019.150047.

SIAFTI. XV Seminário Internacional sobre Atividade Física para Terceira Idade. Anais do Evento. Disponível em https://www.siafticuritiba.com.br/siafti-cwb-20. Acesso em 9 abr. 2021.

UNIVERSIDADE Aberta da Maturidade. Disponível em http://www.uam.ufpr.br/. Acesso em 9 abr. 2021.

VERAS, R. P.; OLIVEIRA, M. Aging in Brazil: The building of a healthcare model. Ciencia e Saude Coletiva, v. 23, n. 6, p. 1929-1936, 2018. Disponível em https://www.scielosp.org/article/csc/2018.v23n6/1929-1936/pt/. Acesso em 11 mar. 2021. https://doi.org/10.1590/1413-81232018236.04722018.

WORLD HEALTH ORGANIZATION. (WHO). Envelhecimento ativo: uma política de saúde. p. 62, 2005. Disponível em https://bvsms.saude.gov.br/bvs/publicacoes/envelhecimento_ativo.pdf. Acesso em 25 mar. 2021.

Recebido em: 16 de Abril de 2021.

Aceito em: 04 de Maio de 2021. 